\title{
NOUVELLES GÉOGRAPHIQUES
}

\section{A NOS LECTEURS}

$\mathrm{E}$ N commençant la deuxième année des Nouvelles géographiques, la rédaction doit tout d'abord remercier les collaborateurs dont le concours :... a permis de mener à bien la première partie de sa tâche. Elle adresse les mêmes remerciements à tous ceux de ses lecteurs, publicistes, membres de l'Université, savants, voyageurs, qui ont bien voulu encourager de leur approbation la Revue naissante, ou l'aider par leurs conseils à se perfectionner et à s'élever.

Ces conseils, accueillis avec reconnaissance, ont été médités et mis à profit. Le format des Nouvelles géographiques sera désormais celui du Tour du Monde; leur périodicité, trop rapprochée pour permettre de donner un développement suffisant aux travaux originaux qui y trouvaient place, sera modifiée à partir de ce jour et deviendra mensuelle, d'hebdomadaire qu'elle était au début. C'est dorénavant avec le premier numéro de chaque mois que le Tour du Monde apportera à ses lecteurs le fascicule de 16 pages in $-4^{0}$ qui remplacera les quatre feuilles séparées contenues dans chaque numéro de l'année dernière.

Tout en restant attachée au Tour du Monde, qui l'a abritée à son début, notre publication répondra au désir qui a été souvent exprimé à ses éditeurs, en prenant une existence propre et en recevant des abonnements indépendants de ceux du Tour $d u$ Monde.

Ges modifications extérieures ne changent rien à l'esprit qui a, dès le premier jour, présidé à la rédaction des Nouvelles géographiques.

Elles continueront à tenir leurs lecteurs au courant du mouvement d'exploration de la terre, non point en rendant exclusivement compte des voyages en pays plus ou moins inconnus, mais en étudiant la science géographique dans toutes ses parties, depuis la physique du globe qui en forme le point de départ, jus- qu'au développement des sociétés humaines, qui en est le point d'arrivée.

Les différents aspects de cette science si vaste et si complexe y seront toujours étudiés avec la plus complète indépendance et la plus absolue bonne foi. Les rédacteurs, se rappelant qu'ils font partie de la phalange en tête de laquelle marchent nos amis Reclus et qui a rénové en France non seulement les méthodes, mais la conception même de la géographie, s'efforceront de garder leur esprit libre de toute idée préconçue et de considérer tout ce qui a trait à la science multiple de la terre comme chose vivante, active, en perpétuel mouvement, et non point comme chose fixée, classée, cataloguée, étiquetée dans un ensemble de notions immuables. Les questions d'enseignement, desquelles dépendent dans une si large mesure l'avenir de la science et la prospérité matérielle de la France, y seront traitées avec la sollicitude qui leur est due. Nos colonies, ces prolongements de la mère patrie dans le monde, de même que les pays où s'exerce l'influence de la France, seront étudiés avec prédilection. Une revue bibliographique, que le format insuffisant de notre première année nous avait obligés à ajourner, tiendra nos lecteurs au courant des principales publications géographiques et cartographiques. De nombreux collaborateurs, membres de l'Institut, explorateurs, professeurs, officiers, savants, publicistes, géographes, consuls ou représentants de la France à l'étranger, nous ont promis ou accordé leur précieux concours dès la première heure, et nous espérons que, grâce à tous ces efforts réunis, la publication qui commence la deuxième année de son existence pourra n'ètre pas inutile aux progrès de la géographie française. Nous nous y emploierons de notre mieux.

\section{Les Éditeurs, la Rédaction.}

